November, 2017

$\mathrm{T}_{\mathrm{E}} \mathrm{X}$ sis 2.17

\title{
Microcanonical Cluster Monte Carlo
}

\author{
Michael Creutz \\ Physics Department \\ Brookhaven National Laboratory \\ Upton, NY 11973 \\ creutz@wind.phy.bnl.gov
}

\begin{abstract}
I propose a numerical simulation algorithm for statistical systems which combines a microcanonical transfer of energy with global changes in clusters of spins. The advantages of the cluster approach near a critical point augment the speed increases associated with multi-spin coding in the microcanonical approach. The method also provides a limited ability to tune the average cluster size.
\end{abstract}

This manuscript has been authored under contract number DE-AC02-76CH00016 with the U.S. Department of Energy. Accordingly, the U.S. Government retains a non-exclusive, royalty-free license to publish or reproduce the published form of this contribution, or allow others to do so, for U.S. Government purposes. 
Monte Carlo simulation is now a major tool for the study of both critical phenomena in condensed matter physics and non-perturbative field theory in particle physics. With continual tuning over the years, current algorithms are well adapted to today's supercomputers. Nevertheless, on large systems near a critical point, severe slowing of the evolution to independent states encourages the search for yet better methods. Two intriguing and apparently unrelated ideas for such improvement are cluster algorithms and microcanonical techniques.

Swendsen and Wang [1] proposed cluster algorithms as a way to make coherent long range changes in a single Monte Carlo updating step. They were motivated by the rather slow probing of important long distance physics by local algorithms when the couplings of a system are near a critical point. Later Wolff [2] presented a powerful variation on the approach, where at each step a single large cluster of variables is formed and modified.

Another useful scheme is the microcanonical Monte Carlo approach [3]. Here a set of additional variables, referred to as demons, are introduced to transfer energy around the system. During the updating, the combined energy of the system of interest and the auxiliary variables is held absolutely constant. In this approach quantities such as the temperature are outputs of the simulation, being extracted from the distribution of demon energies. The primary advantage is that the demons can take a very simple form, and for discrete systems they can be treated entirely with simple bit manipulation. With many demons stored in a few computer words, an effective parallelization is possible on conventional serial computers. In simple cases no floating point arithmetic is ever needed, and many individual spins can be updated in parallel via bitwise operations. This gives programs for the Ising model which run about an order of magnitude faster than conventional approaches. [4]

In this paper I present a combination of these ideas, a cluster algorithm where the cluster growth is determined entirely by bit manipulations involving a set of microcanonical demons. The primary gain is increased simulation speed while retaining the advantages of a global updating scheme near a critical point. In addition, discussions of detailed balance are particularly straightforward in the microcanonical language, simplifying justification of variations in the cluster algorithms. The microcanonical approach also introduces new parameters for tuning the cluster size. As with the local microcanonical method, the 
temperature is determined as a function of the constant total system energy. Wolff [5] has considered another hybrid of the cluster and microcanonical approach, where, rather than the energy, the total area of the cluster boundaries was held fixed. This allowed him to directly and efficiently study the diluted Ising systems of ref. [6].

For simplicity I begin with the simple Ising model. On each site $i$ of an arbitrary lattice lies a spin variable $\sigma_{i}$ taking values in $\{1,-1\}$. The energy of each bond is lowest if the neighboring spins have the same value, and is increased by two units for antiparallel spins. Thus I consider the Hamiltonian

$$
H_{\sigma}=-\sum_{\{i, j\}} \sigma_{i} \sigma_{j}
$$

where $\{i, j\}$ denotes the set of nearest neighbor pairs, each such pair appearing once in the sum. I am interested in the statistical mechanics of this system at inverse temperature $\beta$, and thus consider the partition function

$$
Z_{\sigma}=\sum_{\{\sigma\}} \exp \left(-\beta H_{\sigma}\right) .
$$

As is well known [7] this model has a second order magnetic phase transition at $\beta=$ $(1 / 2) \log (1+\sqrt{2})=0.44068 \ldots$

As in ref. [3] I now augment this system with a set of auxiliary variables called "demons." In that reference the demons were associated with the lattice sites. Here, however, I place them on the system bonds. Each of these demons carries a sack of energy which it can use to "flip" or change the state of the bond it occupies. Thus for each neighboring pair of sites $i$ and $j$, I associate a demon energy $D_{i j} \equiv D_{j i} \geq 0$. For the Ising case the bond energies always change in steps of two, so I am free to restrict the demon energies to be non-negative even integers. In this case it is also convenient to place a capacity limit on the demon in the form of an upper bound for its energy $D_{i j} \leq D_{\max }$. This allows storage of the demon energies in a few bits. For example, with two bits per demon I can consider the individual demon energies to lie in the set $\{0,2,4,6\}$, and store 32 such demons in two 32 bit computer words. As with the local microcanonical approach, the upper limit on $D_{i j}$ still leaves the algorithm exact, although it will modify the cluster shapes. 
The total energy for the coupled system is simply

$$
H=-\sum_{\{i, j\}} \sigma_{i} \sigma_{j}+D_{i j} .
$$

The corresponding canonical partition function is

$$
Z=\sum_{\{\sigma, D\}} \exp (-\beta H) .
$$

This immediately factorizes into contributions from the lattice and the demons. It is only through a microcanonical constraint that these variables become coupled.

The algorithm consists of three parts, cluster growing, cluster flipping, and demon shuffling. For the demon shuffling one merely moves the demons around on the bonds to new locations. This can be done in an arbitrary way, as can easily be seen from the fact that the Hamiltonian in Eq. (3) leaves the demons uncoupled. Indeed, the separate bits of the demons are also uncoupled; thus, the first bits could be shuffled separately from the second bits. (For the test simulations below, the bits were shuffled together.)

For the cluster growing, I divide the demons into two sets, "contented" and "frustrated." A demon is contented if it posesses an amount of energy which allows a change in the state of the currently occupied bond. Thus, if the neighboring spins are parallel and the demon has 2 or more units of energy, then it is happy. If the demon cannot accomodate the change in the bond energy, it is frustrated. This might occur for parallel spins if the demon has no energy, or for antiparallel spins if the demons energy supply is full and cannot accept more. A cluster is now defined as a complete set of sites joined by frustrated demons. All bonds on the exterior boundary of such a cluster carry contented demons. The contentedness of the demon on any given bond corresponds closely with the bond occupation variables used in ref. [6].

I now come to the cluster flipping stage. Here I could either follow the approach of Swendsen and Wang [1] or the variation of Wolff [2]. In the former case, the lattice is divided into clusters as above, and with a random probability all spins in each cluster are either flipped or not. In the Wolff approach, a single random site is chosen, and the corresponding cluster has all of its spins inverted. This picks a given cluster with a probability proportional to its volume, and thus gives larger average cluster sizes. For the 
remainder of this discussion I consider this single cluster approach. When the cluster(s) of spins is(are) flipped, the appropriate changes of the demon energies on the cluster edges must also be made. Thus the energy in Eq. (3) is absolutely conserved.

The justification of the procedure is a particularly simple application of detailed balance. After a cluster is flipped, all demons retain their contented or frustrated state. Thus if we were to regrow the clusters, their shapes would be unchanged. If the demons are not moved and a cluster is grown from the same point, a second application of the algorithm will return exactly to the starting state. With an ensemble in equilibrium, all states of equal energy are equally likely. Under the algorithm the states of the coupled demon-spin system break up into pairs of degenerate states. These states just flip back and forth between each other, and remain equally likely. The demon shuffling stage then changes the cluster breakup for later steps.

As discussed in ref. [3], the microcanonical approach has no explicit coupling parameter in the algorithm. In equilibrium, the temperature is an output and depends on the total system energy. I can easily find it from the distribution of demon energies. Here the separate demon bits are uncoupled; so, I can extract the inverse temperature from the expectation of a single demon bit. For example, I have

$$
\beta=-(1 / 2) \log (-1+1 / P)
$$

where $P$ is the average fraction of the demons with value 2 or 6 . This probability is quickly found by counting the number of times the lowest demon bit is set.

The primary advantage of the approach is that all necessary arithmetic can be done entirely by bitwise operations. For example, on a thirty two bit machine a group of 32 adjacent spins can be stored in a single computer word $I$, and 32 two bit demons can be stored in two computer words $D 1$ and D2. Using shifts and logical operations, the calculations to find the frustrated and contented demons and to grow the cluster can be done for all 32 demons simultaneously. I note in passing that, unlike in the local approaches, no division of the lattice into independent sets of sites, such as a checkerboard, is involved.

The use of bit manipulation gives a potential speed increase over the conventional cluster approach, wherein a bond is included in a cluster based on a floating point comparison with a random number. On the other hand, the gain is not as much as for local algorithms 
because much of the computer time is spent growing the cluster, and once the frustrated bonds are found this is essentially the same in both algorithms. The cluster growth can still be done by bit manipulation, but with a porous cluster much of the work involves irrelevant sites.

Wolff [2] observed that for the Ising model his average cluster size is tied to the magnetic susceptibility. As our demons have upper as well as lower bounds on their energies, this connection is less precise here, with a bond connecting antiparallel spins having an additional chance to be included in the cluster. In general, the clusters tend to be small at high temperatures where it is easy to satisfy the demons desires, while they become increasingly dense at low temperatures where only a few demons will have the required energy to excite their bonds. In Fig. (1) I show the average cluster size as a function of beta for demons carrying from one to four bits. Note how the average cluster size increases as the number of demon bits is reduced. This dependence is essentially invisible with 3 or more bits. Indeed, occupancy of the higher bits is exponentially suppressed by a Boltzmann factor.

Intuitively, the most independent configuration after a single step should be obtained when about half the spins are flipped. In the Ising case this occurs conveniently near the critical temperature. At high temperatures the clusters are quite small, and the algorithm seems to have no advantage over a conventional local approach. Conversely, at low temperatures the clusters dominate the lattice and primarily serve to flip the lattice back and forth between opposite magnetizations. Again, the advantage over a local algorithm is limited. I note, however, that if a hot lattice is rapidly quenched to a low temperature state where several distinct domains are frozen in, the cluster algorithm can easily generate a clusters which fill a single domain. In this case the approach is quite efficient at relaxing the system to its true ground state.

Fig. (1) shows that with single bit demons, for all energies the clusters tend to be quite large, and most spins flip at each step. While the algorithm thus will require more iterations to reach a truly independent configuration, it is perhaps worth noting that in this single bit limit the algorithm is particularly simple. Contented demons, which form the cluster edge, are those where of the three bits consisting of the spins and the demon 
himself an odd number are set. This can be determined by a simple exclusive or operation between the three bits.

Varying the number of demon bits gives some control over the average cluster size. This control could become an essentially continuous parameter because not all demons need have the same number of bits. For simulations of continuous fields, an upper bound on the demon energies similarly will increase the average cluster volume. In general additional constraints on the demon energies work to increase the number of frustrated demons. As it is forbidden to exclude any frustrated demon bonds from the cluster, such constraints will always tend to increase the average cluster size. Unfortunately, it is unclear how to modify the approach to obtain smaller clusters.

In general, cluster algorithms are more limited in their applicability to systems where the spins are continuous variables. The usual approach is to imbed a $Z_{2}$ symmetry in the spin manifold [2]. The microcanonical idea is easily adapted to such systems. In this case, however, the bit manipulation advantages of the discrete models are lost.

To illustrate one way to imbed a $Z_{2}$ transformation in a more complicated model, let me generalize to spins $\sigma_{i}$ taken as elements of a group $G$. For the spin energy associated with a bond connecting sites $i$ and $j \mathrm{I}$ consider $\operatorname{Re} \operatorname{Tr}\left(\sigma_{i}^{\dagger} \sigma_{j}\right)$, where $\operatorname{Tr}$ denotes a group character, perhaps the trace in the fundamental representation. As before, I place auxiliary demon variables on the lattice bonds, with the demon energies now being positive real numbers $D_{i j} \geq 0$.

To keep the detailed balance condition simple, I again arrange transitions between pairs of equal energy states. For the updating of a cluster, I take a random element $g$ of the group to flip the cluster spins around. As the distance from $g$ to some spin $\sigma$ is $g \sigma^{-1}, \mathrm{I}$ perform the flip by multiplying $\sigma$ on the left by the square of this distance, making the new value for the spin $g \sigma^{-1} g$. Note that this transformation has the $Z_{2}$ property of returning to the original spin if repeated twice with the same $g$. Considering such a change on a spin in the partially grown cluster, I determine if the demon on a bond emerging from the cluster has enough energy to make the corresponding change. If not, the demon is frustrated and the adjacent spin joins the cluster.

In Fig. (2) I show the beta dependence of average cluster size for this algorithm as applied in two dimensions to the $\mathrm{U}(1)$ or "X-Y" model, where $\sigma$ is a complex number of 
unit magnitude. At high temperatures the clusters are again small, but for couplings of order unity, where lies the primary physical interest in this model [8], the clusters cover a fair fraction on the system and should give an efficient decorrelation time. For this figure no upper bound was placed on the demon energies; so, the results should be essentially identical to the canonical approach.

The microcanonical approach is easily modified to a canonical method in the same way that the local microcanonical algorithm of ref. [3] reduces to the canonical Metropolis et al. [9] procedure. For this reduction, I refresh the demons after each cluster step; i.e. I replace the demon energies with a random positive numbers selected with Boltzmann

probablity $\exp \left(-\beta D_{i j}\right)$. As for the local case, for large volumes and when the demon scrambling takes place over long distances, the canonical and microcanonical evolutions become essentially indistinguishable. For discrete systems the extra arithmetic involved in going to the canonical scheme may become appreciable.

In summary, I have presented a simulation scheme which combines features of the cluster algorithms of refs. [1],2] with the fast microcanonical approach of ref. [3]. This enables simulations done entirely by bit manipulation, permitting parallel operations on conventional computers. Furthermore, the introduction of the auxiliary demon variables provides a particularly simple way to justify detailed balance for cluster algorithms in general.

\section{ACKNOWLEDGEMENTS}

I thank A. Gocksch, P. Hsieh, Y. Shen, and A. Sokal for useful discussions. 


\section{REFERENCES}

1. R.H. Swendsen and J.-S. Wang Phys. Rev. Lett.프, 86 (1987).

2. U. Wolff, Phys. Rev. Lett.르, 361 (1989).

3. M. Creutz, Phys. Rev. Lett.무, 1411 (1983).

4. M. Creutz, G. Bhanot, and H. Neuberger, Nuclear Physics B235[FS11], 417 (1984); M. Creutz, P. Mitra, and K.J.M. Moriarty, Comp. Phys. Comm. 푸, 361 (1984); M. Creutz, P. Mitra, and K.J.M. Moriarty, J. Stat. Physics $\underline{42}, 823$ (1986); M. Creutz and K.J.M. Moriarty, Comp. Phys. Comm. 쏘, 173 (1986); M. Creutz, K.J.M. Moriarty and M. O'Brien, Comp. Phys. Comm. $\underline{42}, 191$ (1986).

5. U. Wolfe, Nucl. Phys. B300[FS22], 517 (1988).

6. P.W. Kasteleyn and C.M. Fortuin, J. Phys. Soc. Japan 26 (Suppl.), 11 (1969); C.M. Fortuin and P.W. Kasteleyn, Physica 프, 536 (1972); C.M. Fortuin, Physica $\underline{58}, 393$ (1972); C.M. Fortuin, Physica $\underline{59}, 545$ (1972).

7. L. Onsager, Phys. Rev. $\underline{65}, 117$ (1944); T.D. Schultz, D.C. Mattis, and E.H. Lieb, Rev. Mod. Phys. $\underline{36}, 856$ (1964).

8. R. Gupta, J. DeLapp, G.G. Batrouni, G.C. Fox, C.F. Baillie, and J. Apostolakis, Phys. Rev. Lett. $\underline{61}, 1996$ (1988).

9. N. Metropolis, A.W. Rosenbluth, M.N. Rosenbluth, A.H. Teller, and E. Teller, J. Chem. Phys. 21 (1953) 1087. 


\section{Figure Captions}

Fig. 1. The average cluster volume divided by the total system volume as a function of the inverse temperature. The results are for the two dimensional Ising model on a 320 by 320 lattice. The squares, diamonds, crosses, and plus signs are for are for one through four bit demons, respectively.

Fig. 2. The average cluster volume divided by the total volume as a function of the inverse temperature for the $U(1)$ spin model in two dimensions on a 50 by 50 lattice. 